\title{
La estabilidad en el empleo público en la Argentina \\ Triángulo de actores frente a los \\ despidos. Estudio de caso
}

Daniela Alejandra Giordano*

Argentina

\section{Resumen}

En el presente trabajo se analiza la calidad del empleo público considerando la variable de estabilidad, el objetivo de desarrollo sostenible N. ${ }^{\circ} 8$, la vulneración los derechos humanos y prácticas laborales en la Biblioteca Nacional de la República Argentina Mariano Moreno durante el periodo 2008-2015.

\section{Introducción}

El objetivo de este artículo es describir el comportamiento de las organizaciones públicas de la Argentina en relación con la estabilidad en el empleo público y la situación de los contratados frente a los despidos con base en el objetivo de desarrollo sostenible propuesto por la Organización de las Naciones Unidas (ONU) vinculado con la promoción del crecimiento económico sostenido, inclusivo y sostenible, el empleo

\footnotetext{
* Contadora pública. Universidad de Buenos Aires. Especialista en Gerenciamiento
} por Resultados y Control de Gestión. Contacto: dragiordano.dg@gmail.com. 
pleno y productivo y el trabajo decente para todos. Se toma como estudio de caso la Biblioteca Nacional de la República Argentina Mariano Moreno y abarca el período de gestión 2008-2015.

El estudio abarca tres partes. Una primera parte, en la cual se plantea el problema que da origen a la investigación y su justificación. Una segunda parte, en la cual se efectúa un relevamiento acerca de la gestión y evolución del empleo púbico en el organismo. Se parte del encuadre normativo legal aplicable. Luego, se describe la tendencia en la cantidad de personal contratado y se identifican las principales variaciones porcentuales producidas en la categorización del nivel escalafonario. Una tercera parte, en la cual se realiza un análisis acerca de dos materias de responsabilidad social vulneradas, la relacionada con los derechos humanos y aquella vinculada con prácticas laborales. De esta manera, mediante la metodología de estudio de caso, se mencionan algunos de los incumplimientos normativos que generaron derechos humanos vulnerados y luego, se profundiza en otras debilidades vinculadas con la gestión de los recursos humanos. Para finalizar, se presentan las principales conclusiones acerca de la estabilidad laboral en el empleo público de los contratados frente a los despidos y se proponen ejes de acción en los que deberá trabajar la organización.

\section{Responsabilidad social del Estado: garantías constitucionales y la estabilidad en el empleo público}

El concepto del trabajo decente y la calidad del empleo son temas latentes que están en pleno debate internacional tratándose de conceptos dinámicos ya que no significan lo mismo en cualquier contexto. En este punto, la Organización Internacional del Trabajo (OIT) clarifica que la calidad del empleo y el trabajo decente son dos marcos conceptuales distintos, enfatizando en que el establecimiento de mediciones de la calidad del empleo abarca solo algunas dimensiones del trabajo decente (Weller, Roethlisberger, 2011). Sin embargo, se observa que, a pesar de los avances en términos de desarrollo conceptual, la medición de la calidad del empleo o el trabajo decente es todavía un gran desafío. 
El objetivo de este trabajo no pretende desarrollar dichos marcos conceptuales ni la diferencia entre trabajo decente y calidad en el empleo, por lo cual se centra en algunos aspectos que hacen a la calidad del empleo que la amplia bibliografía existente ha desarrollado al respecto. Estos aspectos, entre otros, se refieren a las dimensiones, las variables y los factores determinantes.

Stefano Farné (2002) efectúa un análisis exhaustivo y detallado sobre las variables determinantes de la calidad en el empleo público descritas por once autores, concluyendo en la existencia de una larga lista. Entre las coincidencias de las propuestas además de los beneficios financieros (salariales y no salariales) son importantes los siguientes aspectos: 1) la existencia y el tipo de contrato de colaboración laboral; 2) la seguridad social; 3) las condiciones físicas del puesto de trabajo; 4) las posibilidades de capacitación y el desarrollo profesional; 5) el horario de trabajo y 6) la representación de los intereses de los trabajadores.

Por otra parte, la calidad del empleo según la Comisión Económica de Naciones Unidas para Europa (Unece), las clasifica en once dimensiones. Ellas son: 1) acceso al empleo; 2) trabajo infantil y forzoso; 3 ) ingreso laboral; 4) desarrollo de capacidades y formación continua; 5) cantidad y manejo de horas de trabajo; 6) flexiseguridad; 7) conciliación entre la vida laboral y no-laboral; 8) tratamiento justo en el empleo; 9) trabajo seguro; 10) protección social y 11) diálogo social (Unece, 2007).

Según un informe elaborado por la Comisión Económica para América Latina y el Caribe (Cepal), los factores determinantes de la calidad del empleo incluyen aspectos económicos, institucionales y de calidad propiamente dichos (Weller, Roethlisberger, 2011). Los aspectos de calidad propiamente dichos son las características de la mano de obra, la calidad del puesto de trabajo y la calidad del empleo. Donde la calidad del puesto de trabajo incide directamente sobre la calidad del empleo. Los aspectos institucionales hacen referencia a las normas sobre seguridad, salud e higiene en el trabajo que impactan sobre la calidad del puesto de trabajo y las normas sobre relaciones laborales y capacitación que impactan en la calidad del empleo. Los aspectos económicos hacen al contexto productivo (productividad y heterogeneidad) y otros 
factores determinantes de producción que impactan recíprocamente en la calidad del puesto de trabajo y en el empleo.

De esta manera, se considera el marco analítico para el diagnóstico institucional del servicio civil. Siguiendo a Longo (2004) en un sentido amplio, puede entenderse por Servicio Civil (sc) el sistema de gestión del empleo público y los recursos humanos adscritos al servicio de las organizaciones públicas, existente en una realidad nacional determinada.

En esas líneas de acción, este trabajo pretende analizar la calidad en el empleo público en función de la variable de estabilidad considerando la responsabilidad social que tiene el Estado en esta materia. Por un lado, porque el empleo público contiene garantía constitucional de estabilidad y al mismo tiempo resulta ser necesario para la gestión de políticas públicas que la misma Constitución Nacional reconoce como derechos y garantías de todos los ciudadanos.

Los datos disponibles arrojan que durante el período 2008-2015 el empleo público en la Argentina aumentó. Sin embargo, del comportamiento que el mismo Estado ha impartido en materia de empleo público ha demostrado que el gerenciamiento de dichos años no se esmeró en generar condiciones de estabilidad.

Según un informe elaborado por el Centro de Implementación de Políticas Públicas para la equidad y el crecimiento (Cippec), entre 2003 y 2012, el empleo público en la Argentina se incrementó un 71\%, del cual un $52 \%$ se explica por el aumento del empleo en las plantas permanente y transitoria, y, el $48 \%$ restante, a raíz del crecimiento del personal contratado. Sin embargo, para este período el personal contratado creció un $224 \%$ mientras que el personal de planta permanente aumentó solo un 43\% (Pomares, Gasparin, Deleersnyder, 2013).

La ley marco de empleo público en la Argentina establece tres grandes regímenes de empleo: de estabilidad, de contrataciones por tiempo determinado y de prestación de servicios al personal de gabinete de las autoridades superiores. Sin embargo, las modalidades del régimen de contrataciones y planta de gabinete del personal superior ${ }^{1}$ no gozan de

1 Artículo $7^{\circ}$ de la Ley 25164 de empleo público. 
estabilidad. Para adquirirla se debe ingresar por mecanismos de selección y no ser contratado para tareas de carácter transitorias ${ }^{2}{ }^{3}$ Entre la amplia variedad de casos no enmarcados dentro de la estabilidad del empleado público se encuentran: la planta transitoria, diversidad de variedad de contratados transitoriamente, las plantas transitorias de los convenios colectivos regidos por la ley de contrato de trabajo ${ }^{4}$, los contratos de locación de obra o servicios, los consultores (propios o comúnmente utilizados para los préstamos internacionales), los contratos de locación de obra intelectual por el régimen de compras contrataciones, los contratados por entes cooperadores, los contratados por convenios con universidades públicas.

Lo expuesto precedentemente requiere precisar que se entiende por personal contratado según la bibliografía de referencia existente en la Argentina. La ley del estatuto del personal de la Administración Pública Provincial en la Argentina define el personal contratado como aquel cuya relación laboral está regida por un contrato de plazo determinado y presta servicios en forma personal y directa. Además, expresa que dichos contratos deben ser utilizados exclusivamente para realizar trabajos que a juicio de la autoridad no puedan ser ejecutados o no convengan que sean realizados por el personal permanente dada la especialidad de los mismos (artículo $7^{\circ}$ de la Ley 7233). Por otra parte, la ley de empleo público para la Administración Pública Nacional en la Argentina, define al personal contratado como aquel que comprende exclusivamente la prestación de servicios de carácter transitorio o estacionales, no incluidos en las funciones propias del régimen de carrera y que no puedan ser cubiertos por personal de planta permanente (artículo $9^{\circ}$ de la Ley 25164). Así mismo, el decreto reglamentario de la ley de empleo público en la Argentina especifica que dicho personal carece de estabilidad y su designación o contratación puede ser cancelada en cualquier momento mediante decisión fundada (artículo $30^{\circ}$ del Decreto 214/2002).

2 Artículo $8^{\circ}$ y $9^{\circ}$ de la Ley 25164 de empleo público.

3 Artículo $17^{\circ}$ del Decreto 1421/2002 reglamentario de la ley de empleo público.

4 Ley 20744 de contrato de trabajo. 
En esa misma línea, Gordillo (2013) menciona que en la práctica se denomina personal contratado a los agentes que trabajan para la administración pública no desde cargos o funciones permanentes, sino a través de convenios de plazo limitado (uno o dos años), que no los incorporan a la carrera administrativa ni les otorgan estabilidad en sus empleos. En este punto, quedan excluidas las locaciones de obra o de servicios debido a que, si bien se trata de una modalidad de contratación encubierta, las mismas están previstas para la realización de trabajos, que son de naturaleza estacional, transitoria y representan porcentajes menores. En conclusión, no serán objeto del presente análisis. También, sostiene que entre los criterios para distinguir si el personal contratado se rige por el derecho público o privado, debe tenerse en cuenta aspectos como el sometimiento a un horario de trabajo o arancelamiento de un servicio profesional. Sin embargo, esto no significa que la administración pueda contratar servicios profesionales sin un convenio expreso, a la sola presentación de facturas (Gordillo, 2013).

Ante esta situación, se ponen de manifiesto las diferencias entre los empleados de planta permanente respecto a los trabajadores contratados. En el primer caso, gozan de la garantía de estabilidad, es decir, el derecho de conservar el empleo, la jerarquía y el nivel alcanzado (no el cargo). En el segundo caso, se trata de una excepción a la garantía de estabilidad porque la contratación es realizada con fines de especialidad y transitoriedad. En este punto, Gordillo (2013) agrega cuestiones que hacen a la privación de acceso a una carrera administrativa, la exclusión de los regímenes disciplinarios y la carencia de estabilidad. La carencia de estabilidad queda sujeta a que como las relaciones contractuales son por tiempo determinado no se admite la tácita reconducción una vez vencido el plazo; el contrato se puede rescindir en cualquier momento y su extinción anticipada no da derecho a la indemnización.

Por último, y finalizando las ideas expuestas por Gordillo, él sostiene que el derecho a trabajar goza de protección a nivel público, más cuando se evidencian desviaciones de poder (2013). Esto se fundamenta en que, dadas las definiciones existentes y puestas a consideración, gran parte del personal contratado efectúa actividades de carácter permanente que hacen a la actividad esencial del organismo y no fueron 
contratados exclusivamente para la prestación de servicios de carácter transitorio o estacional. De esta manera, se están cubriendo cargos de planta permanente por contratos de tiempo determinado.

La doctrina y la jurisprudencia también se han manifestado en estos aspectos a través de diferentes fallos. Resulta necesario distinguir los precedentes que sentaron los dictados de tres causas.

Por un largo tiempo y con las bases sentadas en el caso Gil, Carlos Rafael (Corte Suprema, 1989), la doctrina no analizaba si se trataba de tareas eventuales, o si, por el contrario, eran las típicas del personal de planta permanente. En esa sentencia, se admite la excepcionalidad de estos contratos excluyéndolos de la garantía de estabilidad y cargando sobre los trabajadores la obtención de elementos para adquirirla.

Luego la Corte Suprema de Justicia de la Nación cambió de postura y la nueva doctrina es positiva, pero con diferencias sustanciales. Bajo esta nueva doctrina, se distinguen dos sentencias de la Corte Suprema de Justicia de la Nación. Matías Cremonte (2015) sostiene que en caso de tratarse de trabajadores ilegalmente contratados el daño producido al trabajador debe repararse mediante el pago de una indemnización. Por el contrario, cuando se trata de organismos públicos que se rigen por la ley de contrato de trabajo, en virtud de lo cual se despide sin causa a un trabajador ${ }^{5}$, la Corte entiende que el daño se repara mediante la reinstalación. Esta diferencia puede observase en los casos de Madorrán, Marta Cristina (Corte Suprema, 2007) y el caso de Ramos, José Luis (Corte Suprema, 2010). En el primero, la corte reconoce la estabilidad de permanecer en el empleo y prohíbe la ruptura discrecional del vínculo de empleo público, afirmando que la estabilidad no es disponible para las partes. Hace énfasis en el fortalecimiento de los derechos humanos y la dignidad del trabajo, focalizándose en la persona humana. En el segundo, se hace mención al caso Gil, Carlos Rafael y afirma que el paso del tiempo no le otorga derechos, pero reconoce el accionar ilegítimo del Estado y para repararlo fija por analogía la indemnización.

5 Por aplicación del Artículo $245^{\circ}$ de la Ley de Contrato de Trabajo 20744. 


\section{Estudio de caso: Camino hacia una desvalorización del empleo público}

La gestión política de la Biblioteca Nacional, Mariano Moreno, de la República Argentina durante el período 2008-2015, se caracteriza por un aumento sostenido del empleo público necesario para poder cumplir con la misión organizacional y ampliar los ejes de acción. Se entiende por ejes de acción a los objetivos estratégicos en lo que estuvo enfocada tanto en materia de gestión bibliotecaria como de gestión de políticas culturales. De esto, surge que el recurso primario y fundamental en un organismo con estas características es el humano. Profundizado el análisis se desprende que el personal se encuentra vinculado a la organización a través de diversas modalidades de contratación contempladas en la normativa mencionada: planta permanente, plantas transitorias y contratadas.

En particular y analizando el caso de la Biblioteca Nacional, la relación laboral se rige por la Ley de Empleo Público 25164 (в.O.:24/09/1999), el Decreto 1421/2002 (B.O.: 09/08/2002) reglamentario de la ley de empleo público. Dicho decreto es complementado por la Resolución sGP 48/2002 de la Subsecretaria de Gestión Pública (в.O.: 09/01/2003) que establece las pautas para la aplicación del régimen de contrataciones de personal. El convenio colectivo general aplicable es el homologado por el Decreto 214/2006 (в.O.: 01/03/2006) para la Administración Publica Nacional. Así mismo, si bien se trata de un organismo descentralizado dependiente del Ministerio de Cultura tiene autonomía financiera y legal para tener escalafón salarial propio, se rige por el Convenio Colectivo Sectorial Sinep, homologado por el Decreto 2098/2008 (B.O.: 05/12/2008).

En este punto, se observa que, en un lapso de ocho años, la cantidad de personal en el organismo creció en un 227,65\% (de 387 empleados registrados en el año 2008 pasaron a ser 881 registrados en el año 2015). Por otro lado, si consideramos únicamente los últimos tres años que fueron puestos a revisión a fines del año 2015 con el cambio 
de autoridades políticas en la Argentina ${ }^{6}$, la cantidad de personal aumentó en un 189,06\% (de 466 empleados registrados en el 2012 pasaron a ser 881 registrados en el año 2015). Del cálculo anterior quedaron excluidos el personal fuera de nivel y el personal docente por no encontrarse comprendidos dentro de dicho escalafón salarial. El cargo de las autoridades superiores y auditor no sufrieron alternaciones. Por otra parte, el personal docente creció en un $185 \%$ pero considerando los últimos tres años el crecimiento fue de un 17,65\%. Estudiando en profundidad la curva de contratación se desprende que el aumento comienza a darse a partir del año 2010, manteniéndose dentro de rangos normales hasta el 2015.

Entrando un poco más en detalle y en la modalidad de contratación mayormente utilizada surge que a diciembre de 2015 existen ochocientos sesenta y un (861) agentes contratados (artículo $9^{\circ}$ del Decreto 1421/2002); dieciséis (16) agentes de planta permanente y cinco (5) agentes de planta transitoria. Esta situación también queda evidenciada en la formulación presupuestaria del organismo, a través de la cual se desprende que en el rubro "Gastos en Personal”, el 77\% de gastos presupuestados se encuentra destinado para financiar gastos en personal contratado, mientras que el porcentaje de gastos destinados a personal de planta permanente representa tan solo un $10 \%$. En ese sentido, la mayoría de los cargos de planta permanente y transitoria se encuentran vacantes, convirtiéndose la figura del contratado en la modalidad contractual de uso frecuente.

La categorización en los niveles escalafonarios también sufrió alteraciones durante el período analizado. Se entiende por nivel escalafonario a letra aplicable según lo normado por el Convenio Colectivo Sectorial Sinep ${ }^{7}$ para la Administración Publica Nacional aplicable para el pago de la remuneración de los empleados públicos.

Las variaciones producidas en los niveles escalafonarios de los empleados públicos muestran que durante el período 2008-2009 los niveles correspondientes a la letra C y D lideran la escala salarial en

\footnotetext{
6 Artículo $5^{\circ}$ del Decreto 254/2015.

7 Artículo $13^{\circ}$ de Decreto 2098/2008.
} 
proporciones casi iguales del $45 \%$ del total de los empleados. Luego, en el año 2010 el nivel correspondiente a la letra C comienza a tener mayor predominancia, representando el 53,7\% del total de los empleados. Sin embargo, en el año 2011 se da un nuevo giro en la categorización del empleado en el nivel B, convirtiéndose en letra predominante durante el período 2012-2015. De esta manera, culmina el año 2015 con un porcentaje del $83,65 \%$ en el nivel $\mathrm{B}$ e iniciándose los primeros aumentos en el nivel A.

La variación porcentual dentro de cada nivel muestra que, durante el transcurso de los últimos tres años, el nivel correspondiente a la letra B creció en un $141 \%$ mientras que el nivel correspondiente a la letra C, $\mathrm{D}$ y E disminuyeron en un 24 y $50 \%$, respectivamente. Con respecto al nivel correspondiente a la letra A, se ve un aumento del $122 \%$. Por último, si tomamos el período completo 2008-2015 los porcentajes son elevadísimos y van en la misma dirección: aumento sostenido en letra B y A, seguidas de disminuciones en la letra C, D y E.

Ahora bien, la importancia de analizar en términos cuantificables esta modalidad de contratación se fundamenta en dos factores: a) los abusos de poder e incumplimientos normativos y, b) la privación de ciertos derechos y beneficios al personal contratado. En los párrafos siguientes, se sintetizan dichos factores en la Biblioteca Nacional.

Los aspectos normativos que sufren alteraciones son aquellos normados por la ley de empleo público, su decreto reglamentario y el Convenio Colectivo General para la Administración Pública Nacional en la Argentina donde se establece que: 1) el régimen de contrataciones de personal es exclusivamente para la prestación de servicios de carácter transitorio o estacional; 2) la cantidad de personal contratado no debe superar el porcentaje establecido en convenio colectivo de trabajo, o sea el $15 \%$, para el personal de planta permanente; 3) el personal sea equiparado en los niveles y grados del régimen del personal de planta permanente; 4) los perfiles y requisitos del personal a contratar se encuentren definidos con carácter previo a la contratación; 5) el contrato contenga como mínimo las funciones objeto de la contratación, los resultados a obtener; la equiparación escalafonaria que corresponda según los requisitos mínimos para cada nivel escalafonaria y plazo de duración; 6) las excepciones a los requisitos se encuentren autorizadas 
por la Jefatura de Gabinete de Ministros a requerimiento del titular del organismo mediante decisión fundada.

En el caso particular de la Biblioteca Nacional surge que la cantidad de personal contratado supera el $15 \%$ del personal de planta permanente. Así mismo, el personal contratado carece del contrato vigente actualizado y firmado por autoridad competente por lo cual es imposible verificar: a) las funciones objeto de la contratación, b) los resultados obtenidos, c) la equiparación escalafonaria que corresponda, d) el plazo de duración. Además, los perfiles y los requisitos que debe acreditar el personal contratado, en ninguno de los casos fueron realizados con carácter previo a la contratación (artículo $9^{\circ}$ de la Ley 25164 y del Decreto 1421/2002).

Tampoco fueron gestionadas mediante decisión fundamentada y a requerimiento del titular de la Biblioteca Nacional ante la Jefatura de Gabinete de Ministros las excepciones a la equiparación escalafonaria que corresponda según los requisitos mínimos que corresponden para cada nivel escalafonario en los casos de funciones que posean una especialidad crítica en el mercado laboral (artículo $9^{\circ}$, inciso f. del Decreto 1421/2002).

Los derechos y beneficios que les fueron privados, además de aquel vinculado con la estabilidad en el empleo público, son muchos y variados. Los mismos, hacen referencia a cuestiones relacionadas con la gestión de la compensación, los incentivos, la promoción de la carrera y la formación.

Los agentes contratados, si bien pueden realizar los cursos de capacitación ofrecidos por el Instituto Nacional de la Administración Publica (INAP) y obtener un puntaje, pero que al no existir una carrera administrativa establecida en los mismos términos que para el personal de planta permanente, tal puntaje alcanzado pierde todo sentido de existencia. De esta manera, la promoción de grado y tramo queda sujeta al corrimiento en relación con la antigüedad que posee el empleado y no en relación a la capacitación.

Tampoco se les otorgó el derecho de acceder a cargos jerárquicos y estabilidad en el empleo público través de concursos. Esto se debe a que durante más de ocho años no fueron promovidos concursos públicos que permitan acceder a los cargos jerárquicos, que por lo general 
están preservados para autoridades políticas. Así mismo, no se pudo avanzar hacia cargos de mayor relevancia dentro de la organización como consecuencia de lo que se mencionaba: no existe la posibilidad de concursar para acceder a ellos. A lo anterior debe sumarse que aquellos empleados que actualmente desarrollan tareas de jefatura carecen del derecho de cobrar los adicionales por jefatura o función ejecutiva que dispone la normativa vigente para el personal de planta permanente por no estar creados y financiados los cargos según los procedimientos que rigen en la materia.

En síntesis, se puede resumir que estas son las principales debilidades identificadas. Las mismas, se corresponden con el bajo grado de estabilidad laboral, la baja capacitación y profesionalización del personal, la ausencia aperturas de niveles, los deficientes procedimientos de selección de personal, la carencia de sistema de incentivo y las diferencias económicas contractuales producto del abuso de la modalidad de contratación utilizada.

\section{Reflexiones finales}

Estamos frente a un triángulo de actores: los empleados, los políticos (gobernantes y opositores) y los sindicatos que con sus diversas medidas impactan en la calidad del empleo público. El panorama actual muestra tres escenarios. El primero, una gestión política que aumentó el empleo público, pero se fue dejando una situación de precarización laboral desprotegiendo al empleado y por consiguiente su estabilidad debido al exceso y abuso en la modalidad de contratación empleada. El segundo, una nueva gestión gobernante que pretende reordenar y regularizar la situación de precarización laboral heredada comenzando con la adopción de las siguientes medidas: la revisión de las contrataciones de personal y de los procesos concursales efectuados durante los últimos dos y tres años respectivamente de la gestión anterior; la extinción de contratos de trabajo sustentada en el exceso de personal obrante en las organizaciones públicas; la existencia de contratos ficticios; el abuso de contrataciones precarias como los contratos de pasantía y aquellos efectuados con universidades. El tercero, dirigentes 
sindicales que apoyaron el aumento del empleo público a través de contrataciones precarias. Se trata de sindicatos que esperaron ocho años para actuar y reconocer mediáticamente la situación de precarización laboral.

Todos esos casos reflejan situaciones en las cuales se apartaron de los conceptos que hacen a la calidad del empleo público que engloba entre otros aspectos el cuidado de la carrera y su estabilidad.

Ahora, surge la pregunta acerca de cuál es la vía más adecuada para hacer frente a esta situación. ¿Acaso son los despidos la única solución frente a este problema? En mi opinión, no. En este punto, considero que el personal contratado se encuentra alcanzado por la estabilidad de trabajo garantizada en el artículo $14^{\circ}$ bis de la Constitución Nacional, debido a que le fueron vulnerados sus derechos y más cuando se evidenciaron desviaciones de poder por parte Estado.

En este panorama, resulta esencial que el triángulo de actores dialogue y actúe coordinadamente para resolver el tema que les atañe. Las organizaciones públicas deberán crear las condiciones necesarias para que las personas accedan a empleos de calidad que garanticen el trabajo decente de los empleados públicos, así como también proteger los derechos laborales y promover un entorno de trabajo seguro y protegido. Esto se debe a que las organizaciones como parte del Estado tienen el deber y responsabilidad de respetar, proteger, cumplir y hacer realidad los derechos humanos.

Por otra parte, se enfatiza en que la nueva administración contrarreste los efectos del cúmulo de malas decisiones con un análisis metódico y concienzudo de la fuerza laboral que tienda a mantener y desarrollar los recursos con los que se cuenta actualmente. Se aspira a una gestión que analice a través de sus representantes en cada organismo público las contrataciones de personal oportunamente realizadas siguiendo las pautas que ellos mismos establecieron. Se pretende una actuación rigurosa, en aquellos casos en los que se detecten contratos ficticios pero que simultáneamente establezca líneas concretas de acción para una administración óptima y eficiente del capital humano a través de brindar estabilidad al empleado público, promover el desarrollo de la carrera profesional y sus competencias. Se espera de una gestión que, en aquellos casos en los cuales se detecten excesos de 
Responsabilidad Social de las Organizaciones (RSO)

personal, no se los despida inmediatamente, sino que efectúen capacitaciones y las reubicaciones que sean necesarias. Se desea una gestión que valorice a la persona, el derecho de trabajar y la dignidad.

Esto abre un panorama de interminables debates, pero cualquier medida que se tome, al final del camino, tiene impacto en la persona y en la estabilidad del país y la economía. 


\section{Referencias}

Burrell, G., y Morgan, G. (1980). Paradigms, metaphors and puzzle solving in organization theory. Administrative Science Quarterl, 25(4), 605-622. Johnson Graduate School of Management: Cornell University. Recuperado de http://erlanbakiev.weebly.com/uploads/1/0/8/3/10833829/2392283.pdf

Corte Suprema de Justicia de la Nación. (28 de febrero de 1989). Gil, Carlos Rafael c/Universidad Tecnológica Nacional s/nulidad de acto administrativo, indemnización, daños y perjuicios. G 242, XXII.

Corte Suprema de Justicia de la Nación. (3 de mayo de 2007). Madorrán, Marta Cristina c/Administración Nacional de Aduanas s/reincorporación. M. 1488. XXXVI. T. 330. P. 1989.

Corte Suprema de Justicia de la Nación. (6 de abril de 2010). Ramos, José Luis c/Estado Nacional (Ministerio de Defensa-A.R.A.) s/indemnización por despido. R 354, XLIV.T. 333. P. 311.

Cremonte, M. (28 septiembre de 2015). La doctrina de la Corte Suprema ante el fraude laboral en el empleo público. Recuperado de http://ley.exam10.com/pravo/22924/index.html

Decisión Administrativa 883/2010 de autorizaciones de excepción del Decreto 1421/2002. (22 de diciembre de 2010). Boletín Oficial de la Nación, B.o.: 22/12/2010. Argentina: Jefatura de Gabinete de Ministros.

Decreto 1386/1996 de régimen legal de la Biblioteca Nacional. (9 de diciembre de 1996). Boletín Oficial de la Nación, в.o.; 09/12/1996. Poder Ejecutivo Nacional.

Decreto 2749/2003 de aprobación de estructura organizativa. (23 de enero de 2003). Boletín Oficial de la Nación, B.o.: 23/01/2003. Argentina: Poder Ejecutivo Nacional.

Decreto 272/2007 de aprobación de la estructura organizativa de primer nivel operativo. (30 de septiembre de 2007). Boletín Oficial de la Nación, B.O.: 30/09/2007. Argentina: Poder Ejecutivo Nacional.

Decreto 2011/2012 de modificación del Decreto 272/2007. (26 de octubre de 2012). Boletín oficial de la nación, B.o: 26/10/2012. Argentina: Poder Ejecutivo Nacional.

Decreto 1421/2002 reglamentario de la Ley 25154. (9 de agosto de 2002). Boletín Oficial de la Nación, в.O.: 09/08/2002. Argentina: Poder Ejecutivo Nacional. 
Decreto 2098/2008 de sistema nacional de empleo público (Sinep). Homologación del convenio colectivo y acta acuerdo. (5 de diciembre de 2008). Boletín Oficial de la Nación, B.O.: 05/12/2008. Argentina: Poder Ejecutivo Nacional.

Decreto 254/2015 de revisión de procesos concursales y de selección del personal (29 de diciembre de 2015). Boletín Oficial de la Nación, в.O.: 29/12/2015. Argentina: Poder Ejecutivo Nacional.

Facultad de Derecho y Ciencias Sociales (FDyCS). El empleado contratado. Nuestra Joven Revista Jurídica, 1(1), 1-5. Recuperado de http://www.derecho.unc.edu.ar/njrj/revista-no-1/ jurisprudencia/201cel-empleado-contratado201d/view

Gordillo, A. (2013). Capítulo XIII: Agentes Públicos. En Tratado de derecho administrativo y obras selectas (XIII1-XIII32). (1. ${ }^{\mathrm{a}}$ ed.). Buenos Aires: F.D.A. Recuperado de http://www.gordillo.com/tomo1.html

Harmon, M.M., y Mayer, R.T. (1999). Teoría de la organización para la administración pública. México: Fondo de Cultura Económica - Colegio Nacional de Ciencias Políticas y Administración Pública.

Krieger, M. (2001). Sociología de las organizaciones. Una introducción al comportamiento organizacional. Buenos Aires: Prentice Hall.

Ley 7233 de estatuto del personal de la Administración Pública provincial. Provincia de Córdoba (7 de enero de 1985).

Ley 22140 de régimen jurídico básico de la función pública. (25 de enero de 1980). Boletín Oficial de la Nación, B.o.: 25/01/1980. Argentina: Honorable Congreso de la Nación Argentina.

Ley 24146 de administración financiera y control de administración financiera y de los sistemas de control del sector público nacional. (29 de octubre de 2001). Boletín Oficial de la Nación, B.o.: 29/10/1992. Argentina: Honorable Congreso de la Nación Argentina.

Ley 24185 de convenciones colectivas de trabajo. (21 de diciembre de 1992). Boletín Oficial de la Nación, B.o.: 21/12/1992. Argentina: Honorable Congreso de la Nación Argentina.

Ley 24430, ordena la publicación del texto oficial de la Constitución Nacional Argentina sancionada en 1952 con las reformas de los años 1860, 1866, 1898, 1957 y 1994. (10 de enero de 1995). Boletín Oficial de la Nación, B.o.: 10/01/1995. Argentina: Honorable Congreso de la Nación Argentina. 
Ley 25154 marco de regulación del empleo público Nacional. (8 de octubre de 1999). Boletín Oficial de la Nación, в.O.: 08/10/1999. Argentina: Honorable Congreso de la Nación Argentina.

Ley 25188 de ética en el ejercicio de la función pública. (11 de noviembre de 1991). Boletín Oficial de la Nación, B.O.: 01/11/1991. .Argentina: Honorable Congreso de la Nación Argentina.

Longo, Francisco (febrero de 2004). La calidad de los sistemas de servicio civil en América Latina y el Caribe: una metodología de evaluación. Revista del Clad Reforma y Democracia, 28. Caracas.

Mintzberg, H. (2001). Diseño de organizaciones eficientes. Buenos Aires: Ediciones El Ateneo.

Pomares, J., Gasparin, J., Deleersnyder, D. (Septiembre de 2013). Evolución y distribución del empleo público en el sector público nacional Argentino. Una primera aproximación. (Documento de Trabajo $\mathrm{N}^{\circ}$ 117). Buenos Aires: Cipecc. Recuperado de http://www.cippec.org/documents/10179/51827/117+DT+PyGG+Empleo+publico+2013.pdf/ f3cb4e4b-0a4f-4f7f-b66e-c5849ea05118

Resolución 83/2003 de aprobación de estructura, apertura de primeros niveles operativos. (Septiembre 30 de 2003). Boletín Oficial de la Nación, B.o.: 30/09/2003. Biblioteca Nacional.

Resolución 962/2015 de aprobación de estructura, apertura de primeros niveles operativos. (20 de noviembre de 2015). Argentina: Biblioteca Nacional.

Resolución 48/2002 marco de regulación del empleo público nacional. (9 de enero de 2009). Boletín Oficial de la Nación, в.O.: 09/01/2003. Argentina: Subsecretaría de Gestión Pública.

Resolución 384/2014 de actividades de capacitación obligatoria para el personal ingresante. (19 de agosto de 2014). Argentina: Secretaria de Gabinete y Coordinación Administrativa de la Jefatura de Gabinete de Ministros.

Stefano, F. (Noviembre de 2002). Estudio sobre la calidad de empleo en Colombia. (Cuaderno de trabajo N.․ 3). Bogotá: Universidad Externado de Colombia. Recuperado de www.uexternado.edu.co

Unece (Abril de 2007). General presentation on the availability and relevance on indicators to measure the quality of work based on countries' practices. (Documento de trabajo $\mathrm{N}^{\circ}$ 4). Trabajo presentado en Seminar on the quality of work. Geneva, Switzerland. Recuperado de http://www. unece.org/stats/documents/2007.04.labour.htm 
Responsabilidad Social de las Organizaciones (Rso)

Weller, J., Roethlisberger, C. (Abril de 2011). La calidad del empleo en América Latina. Serie Macroeconómica de Desarrollo, 110. Santiago de Chile: Naciones Unidas, Cepal. Recuperado de http://archivo.cepal.org/ pdfs/2011/S2011956.pdf 